\title{
Long non-coding RNA TUG1 is involved in cell growth and chemoresistance of small cell lung cancer by regulating LIMK $2 b$ via $\mathrm{EZH} 2$
}

Yuchun $\mathrm{Niu}^{1,2+}$, Feng $\mathrm{Ma}^{2+}$, Weimei Huang ${ }^{1}$, Shun Fang ${ }^{1}$, Man $\mathrm{Li}^{1}$, Ting Wei ${ }^{3}$ and Linlang Guo ${ }^{1 *}$

\begin{abstract}
Background: Taurine upregulated gene1 (TUG1) as a 7.1-kb IncRNA, has been shown to play an oncogenic role in various cancers. However, the biological functions of IncRNA TUG1 in small cell lung cancer (SCLC) remain unknown. The aim of this study is to explore the roles of TUG1 in cell growth and chemoresistance of SCLC and its possible molecular mechanism.

Methods: The expression of TUG1 in thirty-three cases of SCLC tissues and SCLC cell line were examined by quantitative RT-PCR (qRT-PCR). The functional roles of TUG1 in SCLC were demonstrated by CCK8 assay, colony formation assay, wound healing assay and transwell assay, flow cytometry analysis and in vivo study through siRNA or shRNA mediated knockdown. Western blot assays were used to evaluate gene and protein expression in cell lines. Chromatin immunoprecipitation (ChIP) and RNA binding protein immunoprecipitation (RIP) were performed to confirm the molecular mechanism of TUG1 involved in cell growth and chemoresistance of small cell lung cancer.

Results: We found that TUG1 was overexpressed in SCLC tissues, and its expression was correlated with the clinical stage and the shorter survival time of SCLC patients. Moreover, downregulation of TUG1 expression could impair cell proliferation and increased cell sensitivity to anticancer drugs both in vitro and in vivo. We also discovered that TUG1 knockdown significantly promoted cell apoptosis and cell cycle arrest, and inhibited cell migration and invasion in vitro. We further demonstrated that TUG1 can regulate the expression of LIMK2b (a splice variant of LIM-kinase 2) via binding with enhancer of zeste homolog 2 (EZH2), and then promoted cell growth and chemoresistance of SCLC.

Conclusions: Together, these results suggested that TUG1 mediates cell growth and chemoresistance of SCLC by regulating LIMK2b via EZH2.
\end{abstract}

Keywords: TUG1, Small cell lung cancer (SCLC), Cell growth, Chemoresistance

\footnotetext{
* Correspondence: linlangg@yahoo.com

${ }^{\dagger}$ Equal contributors

1 Department of Pathology Zhujiang Hospital, Southern Medical University,

253 Gongye Road, Guangzhou 510282, People's Republic of China

Full list of author information is available at the end of the article
} 


\section{Background}

Small-cell lung cancer (SCLC) is a highly lethal malignancy that accounts for $10-15 \%$ of lung cancers [1]. SCLC is characterized by a rapid doubling time, high growth fraction, and early development of widespread metastases [2]. Although the incidence of SCLC is reportedly decreasing over time, 5-year survival rates is still lower than $10 \%[3]$. SCLC is highly sensitive to initial chemotherapy and radiotherapy; however, most patients eventually die of widespread metastasis and rapid development of chemoresistance to chemotherapy $[4,5]$. In addition, though genetic changes have been reported in SCLC [6], the precise molecular mechanisms involved in SCLC development and chemoresistance remain to be fully elucidated.

Recently, research has postulated a class of nonprotein-coding RNAs (ncRNAs) that longer than 200 nucleotides in length, defined as long non-coding RNAs (lncRNAs), participates in cell biological processes and human disease pathogenesis $[7,8]$. IncRNAs are poorly conserved and regulate gene expression at various levels, such as chromatin modification, transcription and posttranscriptional processing $[9,10]$. With more and more studies on lncRNA, some researchers classify lncRNA for five broad categories: sense, antisense, bidirectional, intronic, intergenic; and summarize four known molecular functions of lncRNAs: signal, decoy, guide, and scaffold [11]. Interestingly, increasing evidence suggests that lncRNAs play a important role in tumorigenesis, and their aberrant expression confers tumor initiation, cancer cell growth and apoptosis, chemoresistance, invasion and metastasis [12-14]. For example, promotion of lung cancer metastasis by lncRNA MALAT1 (Metastasis Associated Lung Adenocarcinoma Transcript 1); control of hepatocellular cancer cell growth and apoptosis by MEG3; regulation of oesophageal adenocarcinoma cell proliferation and migration by HNF1A-AS1 [15-17]. In addition, studies showed that the long non-coding RNA HOTTIP promotes gemcitabine resistance by regulating HOXA13 in pancreatic cancer [14]. Our laboratory also reported that lncRNA HOTAIR affects chemoresistance by regulating HOXA1 methylation in SCLC [18]. However, functional roles of IncRNAs in SCLC have not been well documented.

The TUG1 (Taurine upregulated gene) IncRNA, located at chromosome 22q12, was originally identified as a transcript up-regulated by taurine [19]. Recently, accumulating evidence has shown that TUG1 is a negative prognostic factor for osteosarcoma patient survival, and high expression of TUG1 in patients has been correlated with enhanced bladder and esophagus cancer cells proliferation and metastasis [20-22]. In previous study, researcher found TUG1 could induced by $\mathrm{p} 53$, then binds to PRC2, and play a key role in cell-cycle regulation [23].
Some studies have explored that TUG1 may regulate genes expression through binding to PRC2. For instance, TUG1 could regulate the expression of HOXB7 by binding to PRC2, then affects cell proliferation in human non-small cell lung cancer [24]. In gastric cancer, TUG1 epigenetically silencing of $\mathrm{p} 57$ by binding with $\mathrm{PRC} 2$ to regulates cell proliferation [25]. However, little is known about TUG1 in SCLC.

In this study, we attempted to explore the potential involvement of TUG1 in SCLC. We found that TUG1 was upregulated in SCLC tissues than matched adjacent normal tissues and its upregulation is related with poor prognosis. Knockdown of TUG1 impairs proliferation, migration, invasion and induces cell apoptosis and cellcycle arrest of human SCLC cell lines. Moreover, we identify the role of TUG1 in chemoresistance in SCLC cells for the first time. Additionally, we found TUG1 affect cell growth and chemoresistance by regulating LIMK2b expression via binding with EZH2. Taken together, our findings suggest that TUG1 may be a novel potential molecular target for treating SCLC patients.

\section{Results}

The expression of TUG1 increased in SCLC tissues and was associated with clinical stage and survival

To investigate the clinicopathological features of TUG1 expression in SCLC, qRT-PCR was performed in 33 tumor samples from SCLC patients. TUG1 expression level was significantly higher in SCLC tumor tissues than those in normal counterparts $(\mathrm{P}<0.01)$ (Fig. 1a). Table 1 summarizes the correlation between TUG1 expression and clinicopathological parameters of SCLC patients. The data indicated that higher expression of TUG1 in extensive disease-SCLC (ED-SCLC) than in limited disease SCLC (LD-SCLC) $(\mathrm{P}=0.011)$. Specifically, we observed higher expression of TUG1 in smoking patients. Kaplan-meier survival analysis based on TUG1 expression showed that high TUG1 expression was correlated with poorer patient survival (Fig. 1b). However, no significant difference was observed with respect to sex (female and male) and age ( $\leq 62$ years and $>62$ years) in our study. Taken together, these results indicated that TUG1 overexpression in SCLC tissues was correlated with stage and survival of SCLC patients.

TUG1 was upregulated in SCLC cell lines and affected cell proliferation in vitro and in vivo

To further investigate the role of TUG1 in SCLC cells, we evaluated the expression of TUG1 in SCLC cell lines (H69, H69AR, H446, H446DDP) and in the normal bronchial epithelial cell line (16HBE) by qRT- PCR. As shown in Fig. 2a, all SCLC cell lines expressed high levels of TUG1 compared with 16HBE. We then tested whether TUG1 was functionally involved in SCLC cell 

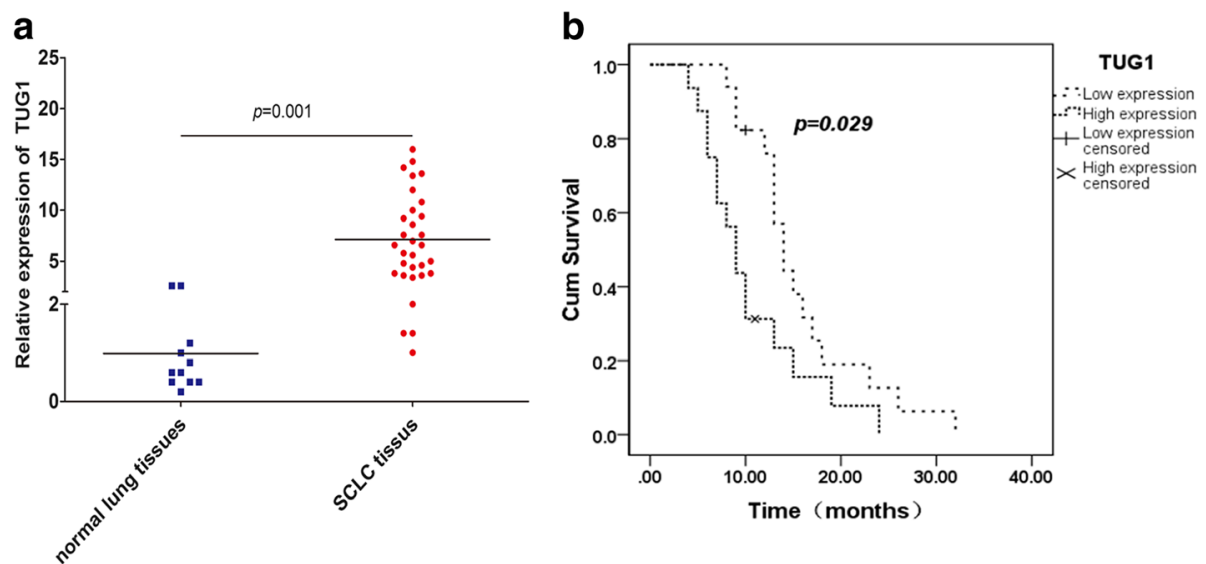

Fig. 1 Relative TUG1 expression and its clinical significance in SCLC tissues. a The expression levels of TUG1 in SCLC tissues $(n=33)$ and adjacent non-tumor tissues $(n=11)$. $\mathbf{b}$ Kaplan-Meier analysis of overall survival of 33 patients with SCLC based on TUG1 expression

growth. We first designed three different TUG1 siRNAs to transfect these four cell lines. qRT-PCR analysis was conducted at $24 \mathrm{~h}$ post-transfection and showed that siTUG1 $1^{*}$ and siTUG1 $2^{*}$ had higher efficiency of interference than siTUG1 $3^{*}$ (Additional file 1: Figure S1 A-D). Then we chose siTUG1 1 and siTUG1 $2 *$ for the following experiments (Fig. 2b). Moreover, we also established stable TUG1 knockdown SCLC cell lines by retrovirus infection (Fig. 2c). CCK-8 assay and colony formation assay were used to detect the effect of TUG1 knockdown on growth of the SCLC cell lines. As shown in Fig. 2d, SCLC cells transfected with siTUG1 showed greatly reduced cell proliferation rate. Similarly, the colony formation assay demonstrated that the number of colonies decreased significantly in SCLC cells transfected with shTUG1 as compared with shControl (Fig. 2e).

The tumorigenic properties of TUG1 in vivo were performed in male nude mouse xenograft. We injected H446 and H69 cells transfected with either shControl or shTUG1 into nude mice. As shown in Fig. 3a-c, downregulation of TUG1 significantly inhibited tumor growth. We also performed qRT-PCR to detect the expression of

Table 1 Association of TUG1 expression and clinicopathological characteristics in 33 SCLC patients

\begin{tabular}{|c|c|c|c|c|c|}
\hline \multirow[t]{2}{*}{ Characteristics } & \multirow{2}{*}{$\begin{array}{l}\text { Total } \\
(n=33)\end{array}$} & \multicolumn{2}{|l|}{ TUG1 expression } & \multirow[t]{2}{*}{$x^{2}$} & \multirow[t]{2}{*}{$P$ value* } \\
\hline & & Low expression & High expression & & \\
\hline Gender & & & & 1.816 & 0.174 \\
\hline Male & 26 & $15(57.7)$ & $11(42.3)$ & & \\
\hline Female & 7 & $2(50.0)$ & $5(50.0)$ & & \\
\hline Age (years) & & & & 0.299 & 0.420 \\
\hline$\leq 62$ year & 19 & $9(47.4)$ & $10(52.6)$ & & \\
\hline$>62$ year & 14 & $8(57.1)$ & $6(42.9)$ & & \\
\hline Smoking history & & & & 3.529 & 0.032 \\
\hline YES & 20 & $7(35.0)$ & $13(65.0)$ & & \\
\hline NO & 13 & $10(76.9)$ & $3(23.08)$ & & \\
\hline Disease stage & & & & 6.651 & 0.011 \\
\hline Limited disease (LD) & 16 & $12(75.0)$ & $4(25.0)$ & & \\
\hline Extensive-stage disease (ED) & 17 & $5(29.4)$ & $12(70.6)$ & & \\
\hline Status & & & & 4.520 & 0.036 \\
\hline Survival & 10 & $8(80.0)$ & $2(20.0)$ & & \\
\hline Death & 23 & $9(39.1)$ & $14(60.9)$ & & \\
\hline
\end{tabular}

*For analysis of correlation between of TUG1 expression levels and clinical features, Fisher's Exact Test were used. Results were considered statistically significant at $P<0.05$ 


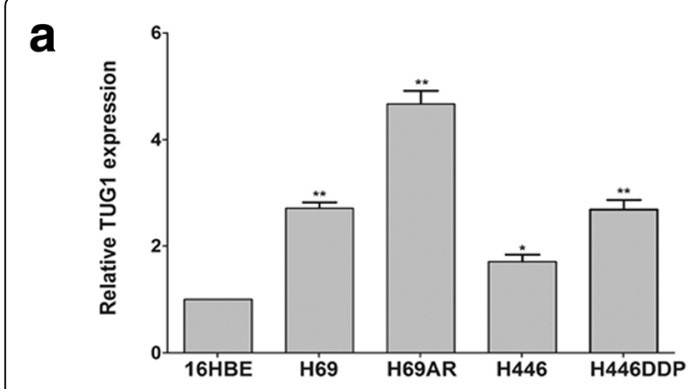

C

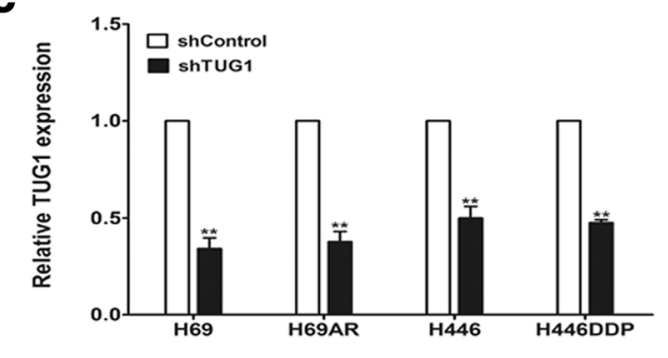

b

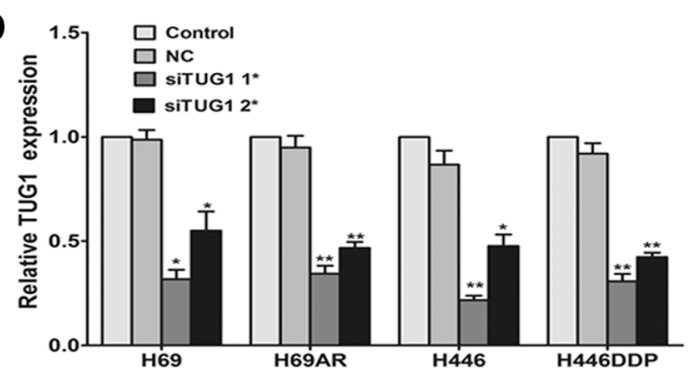

d

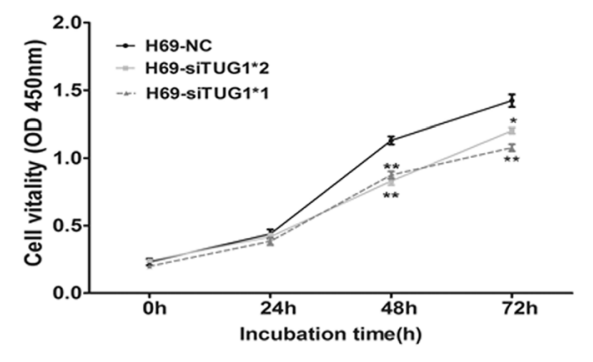

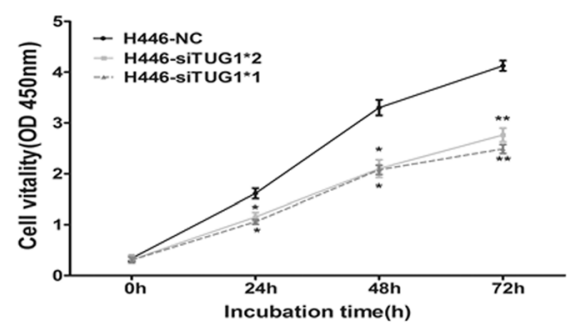
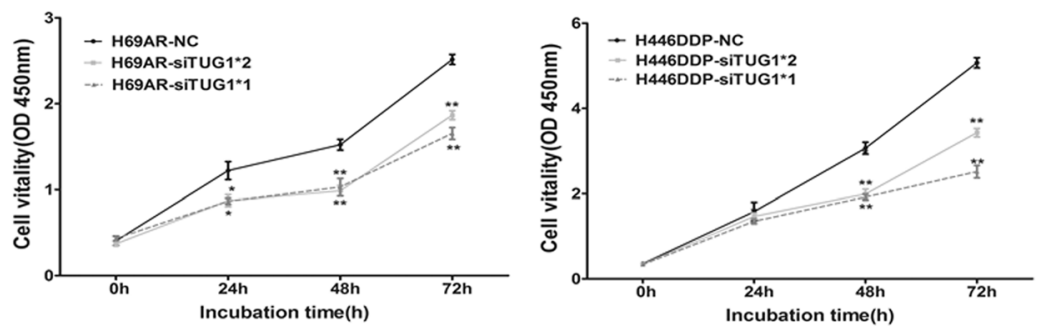

e

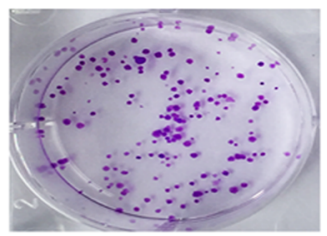

H446-shControl

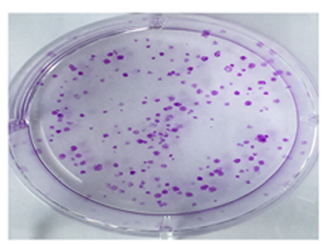

H69AR-shControl

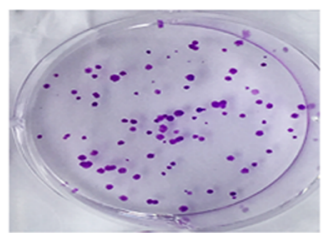

H446-shTUG1

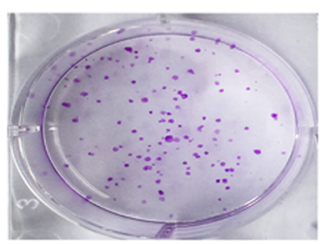

H69AR-shTUG1

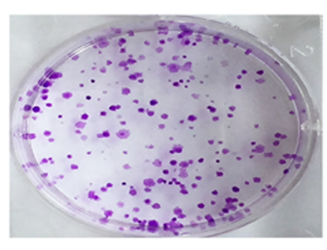

H446DDP-shContro1

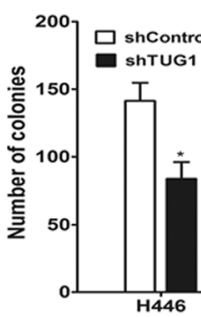

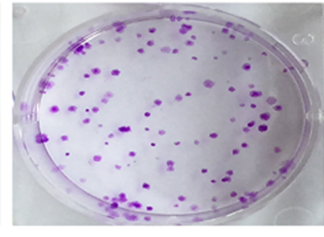

H446DDP-shTUG 1

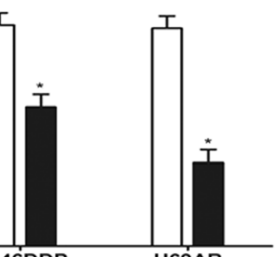

Fig. 2 TUG1 was up-regulated in SCLC cell lines and TUG1 knockdown inhibited cell proliferation in vitro. a The expression of TUG1 was assessed in SCLC cell lines compared with the normal bronchial epithelial cell line (16HBE) by qRT-PCR. $\mathbf{b}$ c Inhibition of TUG1 by transfection of TUG1 SiRNAs or sh RNA in H69、H69AR、H446、H446DDP cells. d CCK-8 proliferation assays were used to determine the cell viability for siTUG1 transfected SCLC cells. Experiments were performed in triplicate. e Colony formation assays were performed to determine the proliferation of shTUG1 transfected H446, H446DDP and H69AR cells. Representative photographs are shown, and the numbers of colonies were counted. ${ }^{*}, P<0.05 ;{ }^{* *}, P<0.001$

TUG1 in tumor tissues selected from mice (Fig. 3d). Results showed that the expression levels of TUG1 in the shTUG1 group were lower than those in the control group. Taken together, these data suggested the TUG1 affects SCLC cell proliferation and growth.

\section{TUG1 involved in SCLC cell migration and invasion}

Wound healing assay and transwell assay were performed to investigate whether TUG1 had a functional role in cell migration and invasion in SCLC. Result of wound healing assay demonstrated the migration 


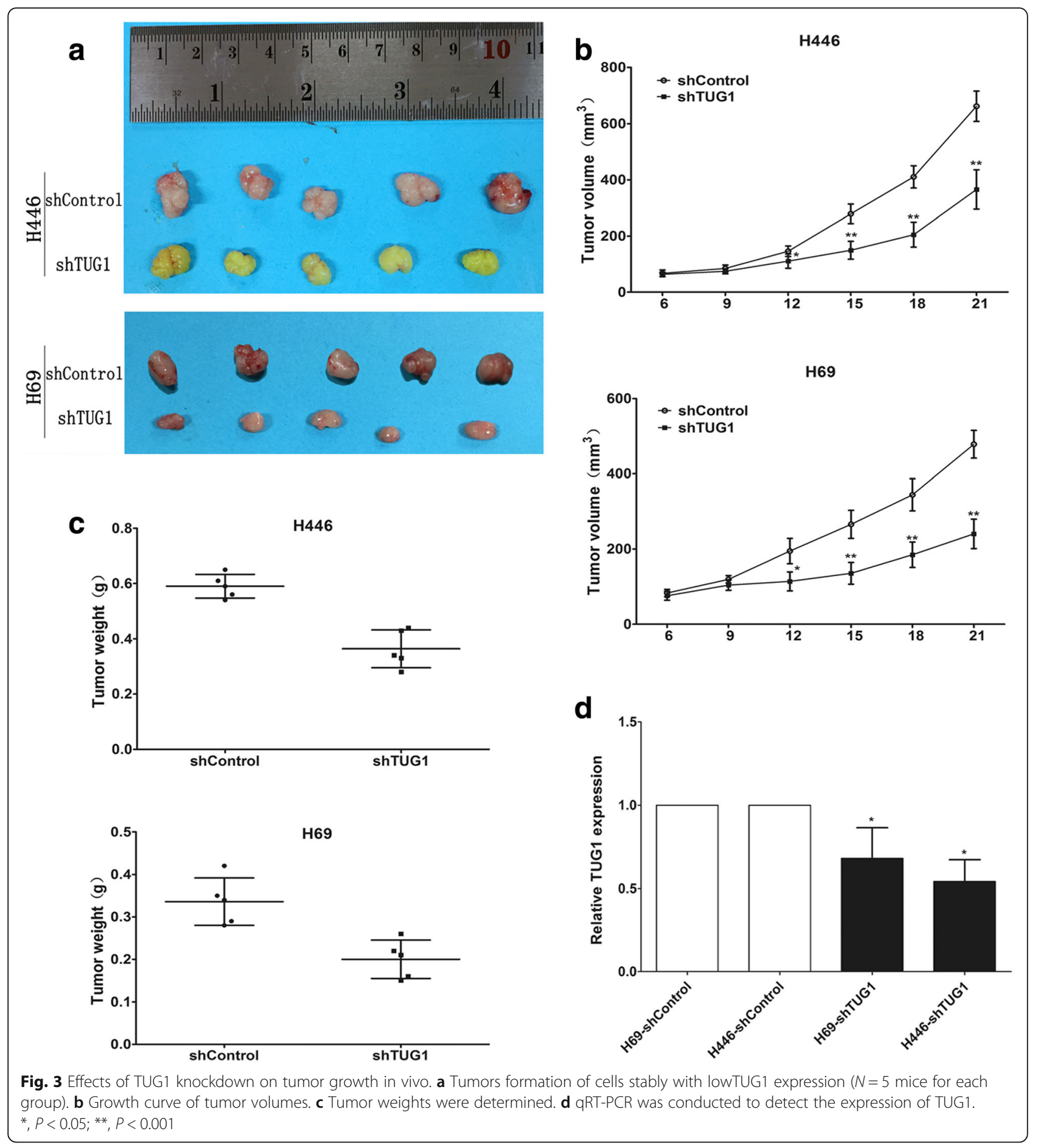

distance of SCLC cells infected by shTUG1 was significantly wider than the shControl group (Additional file 2: Figure S2 A). In the transwell assay, the number of shTUG1 infected cells that migrated through the membrane were significantly less than the shControl group (Additional file 2: Figure S2 B). These results showed that inhibition of TUG1 could significantly impair SCLC cell migration and invasion ability compared with control group.

TUG1 regulated cell apoptosis and cell cycle in SCLC cells To further probe the potential mechanisms underlying the growth-inhibitory effects of TUG1 knockdown, we assessed cell apoptosis and cell-cycle in H446 and H69 
cells. Flow cytometry analysis demonstrated that TUG1 knockdown led to a significant increase of cell apoptosis (P $<0.05)$ and a significant accumulation of cells at G1-phase $(\mathrm{P}<0.001$, Additional file 3: Figure S3 A and B). These data suggested that TUG1 mediated promotion of SCLC cell growth may be mediated by regulation of the apoptosis and G1-phase . Moreover, to investigate the possible mechanisms of TUG1 in chemoresistance, we also conducted flow cytometry analysis to examine the impact of TUG1 on apoptosis when exposed to chemotherapeutic drugs. The results showed that downregulation of TUG1 in H446DDP and H69AR cells resulted in increased drug-induced apoptosis after treatment with ADM, DDP or VP-16 (Additional file 4: Figure S4).

\section{TUG1 expression was associated with SCLC chemosensitivity in vitro and in vivo}

To further investigate the impact of TUG1 on SCLC chemosensitivity, we detected the differential expression of TUG1 in SCLC drug-sensitive cells (H69 and H446) and drug-resistant cells (H69AR andH446DDP) by qRT-PCR. The results showed that TUG1 over-expression in H69AR and H446DDP cells than that in $\mathrm{H} 69$ and H446 cells (Fig. 2a). After knockdown of TUG1, the IC50 values of H446DDP and H69AR cells significantly decreased with treatment of chemotherapeutic drugs including DDP, ADM or VP-16 (Fig. 4a).

We then used a nude mouse xenograft model to further investigate the ability of TUG1 to confer chemoresistance in SCLC. H446DDP cells transfected with shTUG1 or shControl were subcutaneously injected into mice. As shown in Fig. 4b, tumor growth was inhibited in the shTUG1 group treated with PBS or drugs (DDP and VP-16) compared with the controls. Tumor grew significantly more slowly in mice following combined drugs treatment and TUG1 knockdown. Four weeks later, the mean tumor volume for the TUG1-knockdown group and the drugs group was obviously smaller than that of the control group (Fig. 4c). Moreover, combined treatment with TUG1 knockdown and drugs led to an even further reduction in tumor volume. Similarly, the average tumor weight in shTUG1 group combined treatment with drugs showed a similar trend (Fig. 4d). qRT-PCR analysis of TUG1 expression found it to be significantly lower in tumor tissues formed from shTUG1 group than those from controls (Fig. 4e). These results suggested that downregulation of TUG1 increased the in vivo chemosensitivity of H446DDP cells to drugs.

\section{TUG1 affected cell growth and chemoresistance of SCLC by regulating LIMK2b expression}

To determine how TUG1 affects cell growth and chemoresistance of SCLC, we conducted the bioinformatics analysis to identify its potential downstream target genes. We first searched the database and found that
TUG1 was previously identified to have an enhancer-like function and positive influence on the neighboring protein-coding genes [26]. We then predicted 12 TUG1 nearby coding genes (distance $<300 \mathrm{~kb}$ ) including LIMK $2 \mathrm{~b}$ by searching UCSC Genome database (http:// genome.ucsc.edu/). Finally, we confirmed LIMK2b may be the potential target gene in SCLC according to our cDNA microarray analysis in which 1.5-fold upregulation in drug resistance SCLC cells as compared to the parental SCLC cells. We further performed qRT-PCR and Western blot analysis to confirm our supposition. The results showed that knockdown of TUG1 can inhibit LIMK2b expressions both at mRNA and protein levels in H446DDP and H69AR cells (Fig. 5a).

We next examined whether LIMK2b played an important role in TUG1 mediated cell growth and chemoresistance by rescue experiments. Transfection of LIMK2b-GFP in H446DDP and H69AR cells completely reversed the downregulation of LIMK2b induced by TUG1 (Fig. 5a). CCK8 and colony formation assay results suggested that cotransfection can partially rescue shTUG1 impaired proliferation and chemoresistance (Fig. 5c and d). These results indicate that TUG1 promotes SCLC cell proliferation and chemoresistance partly through downregulation of LIMK2b expression.

Subsequently, we detected the relationship of TUG1 and LIMK2b in SCLC FFPE tissues. Consistent with the results obtained from SCLC cell lines, LIMK2b was overexpressed in SCLC tissues and the expression levels of LIMK2b were positively correlated with those of TUG1 by qRT-PCR in 33 SCLC FFPE tissues (Fig. 6a and b).

\section{TUG1 regulated LIMK2b expression by binding with EZH2} On the basis of the study above, we found that TUG1 could regulate the expression of LIMK2b, then we test whether TUG1 has a direct combination with LIMK2b. However, the result of RIP analysis showed no direct combination between TUG1 and LIMK2b (Fig. 6c). TUG1 was previously reported to mediated transcriptional regulation through binding with EZH2 [23-25, 27]. Then we suppose that TUG1 may regulate LIMK2b expression by binding with EZH2. We designed three different EZH2 siRNAs and to transfect H446DDP and H69AR cells (Additional file 1: Figure S1 E-F). To further confirm the results, we also applied EZH2 inhibitors EI1 or GSK343 to treat H446DDP and H69AR cells. The results showed that LIMK2b level was downregulated after EZH2 expressions were inhibited (Fig. 6d and e).

Furthermore, we conducted ChIP assays and found that knockdown of TUG1 decreased the binding of EZH2 and H3K27me3 levels across the LIMK2b promoter compared to cells transfected with siNC (Fig. 6f). Taken together, these data suggest that TUG1 is required to target EZH2 occupancy and activity to epigenetically modulate the expression of LIMK2b. 


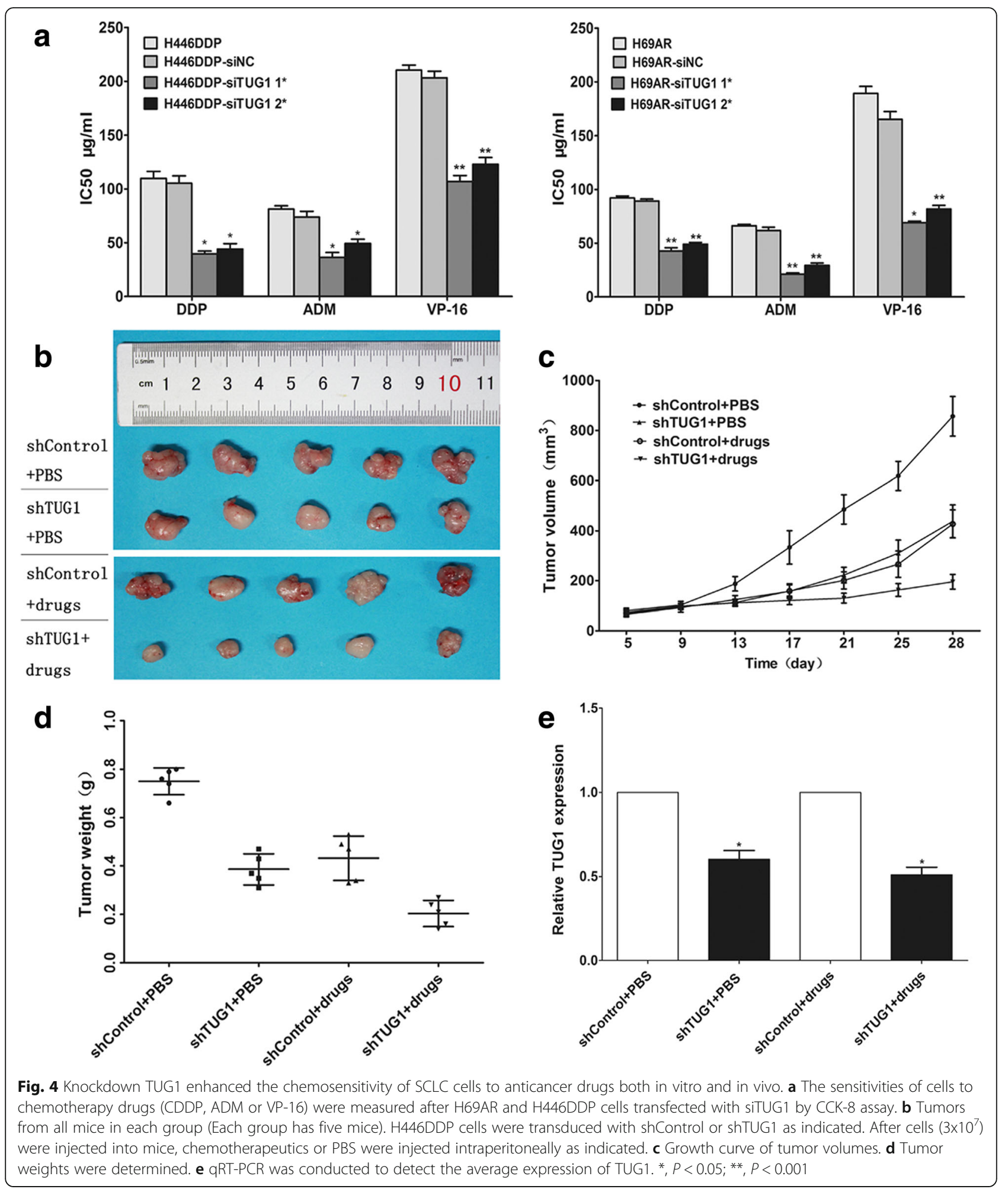

\section{Discussion}

Recently, the study about the biological function of TUG1 has become one of the hottest topics in various cancer. TUG1 was overexpressed in various solid tumor including osteosarcoma, bladder, esophagus, gastric and liver cancer [20-22, 25, 27]. Nonetheless, the clinical features of TUG1 expression in SCLC have not been reported yet. In this study, we analyzed the expression of TUG1 in 33 cases of human SCLC tissues and found that the high expression level of TUG1 indicates shorter 


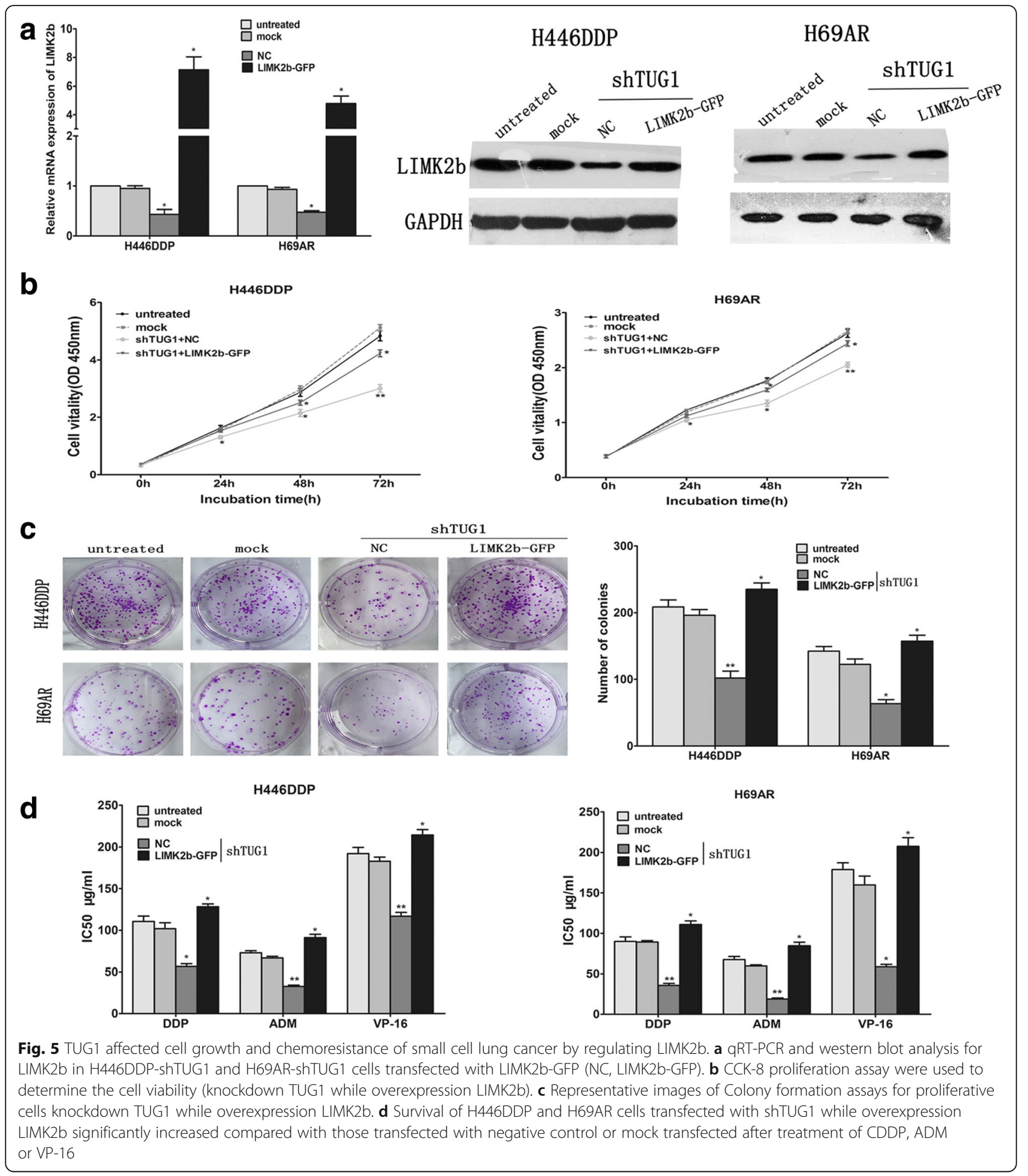

survival time of the SCLC patients. Therefore, our research may provide an independent prognostic factor for SCLC patients.

To explore the functional role of TUG1 in SCLC, we therefore established stable TUG1-downexpressed cells in the study. Our data indicate that TUG1 was upregulated in SCLC, inhibition of TUG1 expression resulted in decreased cell growth and enhanced chemosensitivity both in vitro and in vivo. Moreover, we found that knockdown of TUG1 also increased cell apoptosis, G1 


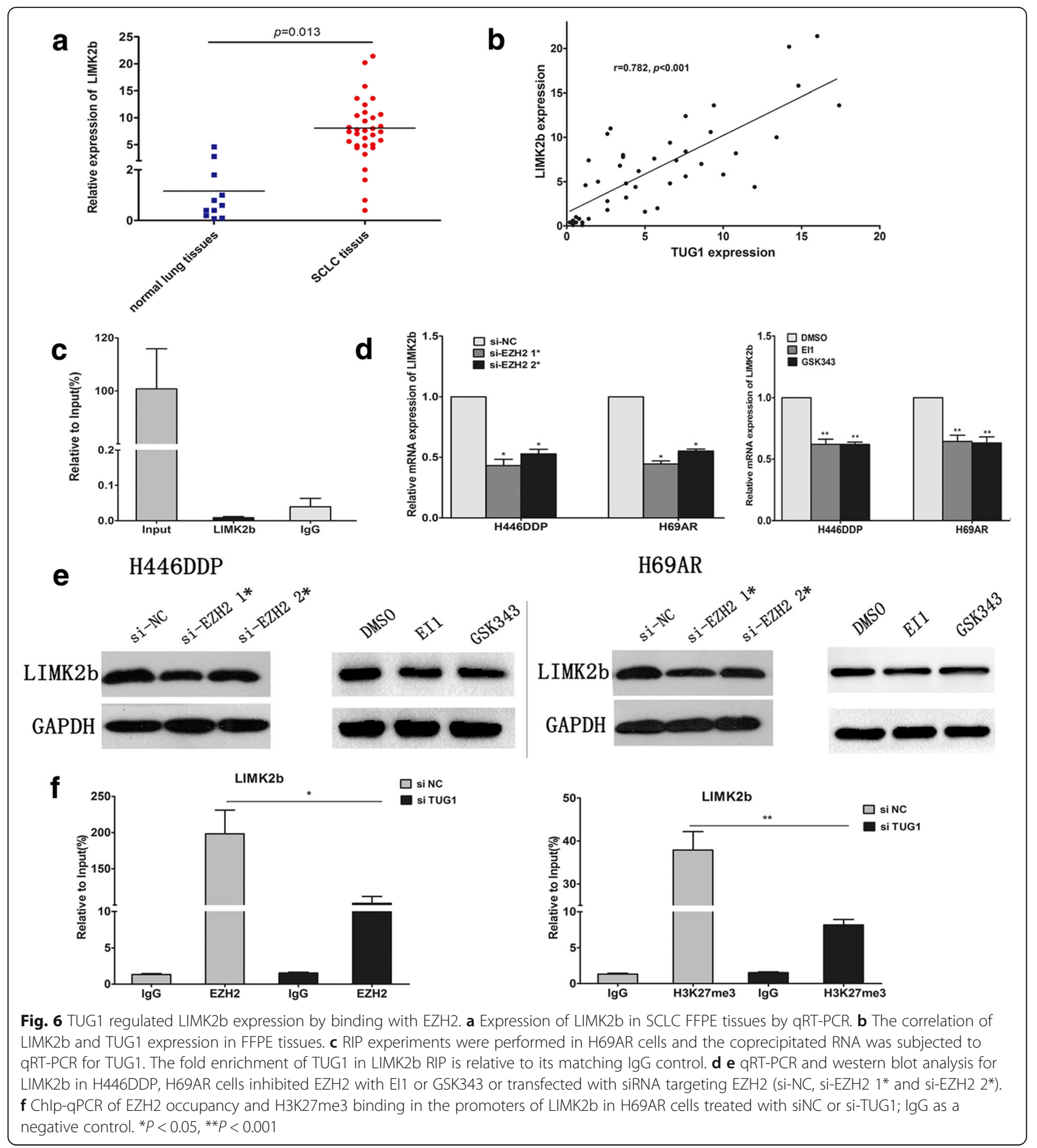

cell-cycle arrest, and impaired SCLC cell migration and invasion ability.

To further investigate the mechanisms of TUG1 involved in cell growth and chemoresistance, we conducted the bioinformatics analysis and found LIMK2b, which located at $300 \mathrm{kp}$ of TUG1. LIMK2 is a member of LIM kinase (LIMK) family that includes LIMK1. LIMK2 encodes a kinase that regulates actin dynamics through phosphorylation of cofilin, and comprises two alternative transcripts, LIMK2a and LIMK2b [28-30]. Recent reports illustrate that LIMK2 is involved in tumor growth, and induces migration and invasion of tumor cells [31-33]. Additionally, studies also showed a negative correlation between LIMK2 and anticancer drugs, which suggesting that LIMK2 may be a predictive marker of drug resistance 
$[34,35]$. Previous study showed that LIMK2b could encode only one and a half LIM domains after the first LIM domain partially replaced, which is special to the LIMK2 gene and conserved between murine and human genes [36, 37]. Recent studies demonstrated that LIMK2b is a direct target of p53 and involved in the control of cell proliferation and cell division. The report also showed that LIMK2b has a critical role in promoting the G2/M DNA-damage checkpoint [38]. In the present study, we firstly hypothesized that TUG1 may associated with LIMK2b. Our results showed that knockdown of TUG1 significantly decrease the expression of LIMK2b. Furthermore, the impacts of TUG1 on cell growth and chemoresistance were reversed by concomitant LIMK2b -GFP, which indicating that the effect of TUG1 on cell growth and chemoresistance is partly mediated through LIMK2b. Moreover, we also found that LIMK2b was over- expressed and positively correlated with TUG1 in SCLC tissues. However, RIP analysis indicated that there was no direct combination between TUG1 and LIMK2b, which suggested that TUG1 affected cell growth and chemoresistance is not directly through LIMK2b. The results stated above raises an interesting question: What is the linker between TUG1 and LIMK2b? Several recent studies indicated that TUG1 regulate genes expression by binding with EZH2 to affect cell proliferation in human non-small cell lung cancer, gastric cancer and hepatocellular carcinoma [24, 25, 27]. So, we hypothesized that EZH2 may be a linker between TUG1 and LIMK2b. To prove our hypothesis, we used ChIP assays to demonstrate that knockdown of TUG1 decreased the binding of EZH2 and H3K27me3 levels across the LIMK2b promoter. EZH2 as an important component of polycomb repressive complex2 (PRC2) has been reported to be necessary for the formation of the H3K27me3, and recruits histone deacetylases then resulting in gene transcriptional repression in cancer cells [39-41]. These results suggested that TUG1 epigenetically regulated LIMK2b through EZH2.

\section{Conclusions}

In summary, our study showed that TUG1 was upregulated in SCLC tissues and its overexpression is closely associated with clinical stage and overall survival in patients with SCLC. Furthermore, the effects of TUG1 on cell proliferation, cell apoptosis, cell cycle regulation, migration, invasion and chemoresistance indicated that TUG1 promotes tumorigenesis. We also demonstrated that TUG1 is involved in cell growth and chemoresistance of SCLC through regulating LIMK2b by binding with EZH2. This study may provide a strategy and lead to the development of lncRNAs directed diagnostics and therapeutics against SCLC.

\section{Methods}

Patients and tissue samples

A total of 33 formalin-fixed, paraffin-embedded (FFPE) tissues (33 primary cancerous, 11 adjacent non cancerous tissues) were collected from patients who had underwent bronchofiberscopy or biopsy for SCLC between the period 2009.1 and 2013.11 and receiving care and follow-up at The First Affiliated Hospital of Hebei North University, informed consent was obtained from all patients before sample collection. The experiments were approved by the Ethics Committee of The First Affiliated Hospital of Hebei North University, and conformed to the standards set by the Declaration of Helsinki. Clinical data included the patient gender, age, smoking history, limited- or extensivestage disease and follow-up (Table 1).

\section{Cell culture and treatment}

Human SCLC cell line NCI-H69, NCI-H446, NCIH69ARwere purchased from the American Type Culture Collection (ATCC, United States of America (USA)) and maintained in RPMI1640 medium contain in L-glutamine with $10 \%$ and $20 \%$ fetal calf serum respectively in an incubator at $37{ }^{\circ} \mathrm{C}$ with $5 \% \mathrm{CO} 2$. The cisplatin-resistant NCI-H446DDP cell line was obtained by culturing cells in gradually increasing doses of cisplatin up to $2.0 \mathrm{uM}$ after a total of 7 months in our laboratory. The drugresistant cells were maintained in drug-free medium for at least 2 weeks before any experiment. To inhibit EZH2 activities, cells were treated with $12 \mu \mathrm{M}$ GSK343 (S7164, Selleck) or $10 \mu \mathrm{M}$ EI1 (S7611, Selleck) for $36 \mathrm{~h}$.

\section{RNA isolation, quantitative reverse transcription- polymerase chain reaction (qRT- PCR)}

Total RNA was extracted from cell lines and FFPE tissues using TRIzol (Invitrogen), RNeasy FFPE Kit (Qiagen) according to the manufacturer's instructions. According to Prime Script RT reagent Kit (TIANGEN, Beijing, China), reverse transcription reactions were processed at $42{ }^{\circ} \mathrm{C}$ for $15 \mathrm{~min}$, followed by $3 \mathrm{~min}$ at $95^{\circ}$ $\mathrm{C}$ for cDNA synthesis. Then quantitative real time PCR was performed in an ABI illumina instrument. Primers were designed by Shanghai Sangon Biotech Co Ltd. TUG1 F: 5' TAGCAGTTCCCCAATCCTTG3'; R: 5' CACAAATTCCCATCAT TCC- C3'; LIMK2b F: 5' AGGCAGTCACAGACGGATTT3'; R: 5'GAGCTTCC CATCCT- TCTCATAG 3'; GAPDH was used as an endogenous control. The relative gene expression levels of TUG1 were determined using the comparative delta-delta CT method $\left(2^{-\Delta \Delta C t}\right)$. 


\section{Cell transfection}

SCLC cells were transiently transfected with small interfering siRNA or scrambled siRNA negative control (NC) . Following the manufacturer's protocols, cells were seeded in six-well plates and transfected with siRNA by Lipofectamine 2000 (Invitrogen) when grew to reach about 70\% confluence. Three individual TUG1 siRNAs (siTUG1 1*, siTUG1 $2 \%$, siTUG1 $3 * 1$ ), EZH2 siRNA (si-EZH2 $1 *$, siEZH2 $2^{*}$, si-EZH2 $3^{*}$ ) and siNC were designed by GenePharma Inc (Shanghai, China). The nucleotide sequences of siRNAs for TUG1 and EZH2 were shown in table S1.

The lentiviral particles of shTUG1 (forward, 5' GATCCGCTTGGCTTCTATTCTG AATCCTTTCAAGA GAAGGATTCAGAATAGAAAGCCAAGCCAAGCTTT TTTG-3' and reverse, 5'-GCGAACCGAAGATAA GACTTAGGAAAGTTCTCTTCCTAA GTCT TATC TTCGGTTCGAAAAAAC-3') and LIMK2b-GFP were also designed and purchased from GenePharma Co., Ltd. To generate the lenti-viruses, shRNA plasmids were cotransfected into SCLC cells along with envelope (VSVG) and packaging (pGag/Pol, pRev) plasmids using lipofectamine 2000 (Invitrogen). The viral supernatants were harvested and filtered after $48 \mathrm{~h}$ transfection. Cells were infected in the presence of a serum-containing medium supplemented with $8 \mu \mathrm{g} / \mathrm{ml}$ polybrene. Following infection for $48 \mathrm{~h}$, cells were selected with $2.0 \mu \mathrm{g} / \mathrm{ml}$ puromycin (Sigma). Knockdown efficiencies were examined by qRT-PCR.

\section{Cell counting kit-8 (CCK-8) assay}

Cell proliferation and drug resistance were assayed by the Cell Counting Kit-8 (CCK8) assay. For cell proliferation assay, transient transfection cells were seeded in 96-well plates about $5 \times 10^{3}$ cells per well. According to the manufacturer's protocol, testing cell proliferation every $24 \mathrm{~h}$. For cell drug resistance assay, after transient transfection cells, then treated it with drugs for $24 \mathrm{~h}$. Three chemotherapy drugs [Cisplatin (DDP; Shandong, China), Adriamycin (ADM; Jiangsu, China) Etoposide (VP-16; Jiangsu, China),] were used. After incubation with 10ul of CCK-8 reagent (Beyotime Institute of Biotechnology, shanghai, China) for $2 \mathrm{~h}$ or $4 \mathrm{~h}$, the absorbance at $450 \mathrm{~nm}$ was measured. The cells incubated without drugs were set at $100 \%$ survival and were used to calculate the concentration of each chemotherapeutic drug IC50. The assay was performed in five replicate wells, and three parallel experiments for each sample were conducted.

\section{Colony formation assay}

Collected cells that transduced with shTUG1, both shTUG1 and LIMK2b-GFP or control shRNA and seeded (200 cells/well) in six-well plates. Then, the cells were incubated in an incubator with $5 \% \mathrm{CO} 2$ at $37{ }^{\circ} \mathrm{C}$. After two weeks later, removed the culture medium, and rinsed cells three times with PBS. Nextly, the cells were fixed with $4 \%$ paraformaldehyde, then stained with $0.1 \%$ crystal violet. The number of colonies were counted by visual inspection.

\section{Cell invasion and migration assay}

For the invasion assays, 24-well Matrigel invasion chambers

(Corning Incorporated, Corning, NY, USA) was used. After TUG1 knockdown, $3 \times 10^{4}$ cells were seeded on the upper chamber. To stimulate invasion, the bottom chamber was added $500 \mu \mathrm{L}$ medium with $20 \%$ FBS . After $48 \mathrm{~h}$, cells in the bottom chamber were stained with $0.1 \%$ crystal violet, then counted using a microscopy $(100 \times$ magnification). Additionally, Wound healing assay was performed for analysis of cell migration. Cells transfected with either shTUG1 or shNC, were seeded on six-well plates, then created an artificial scratch wound with a $100-\mu l$ pipette tip. Cells with serum-free medium for a further 24-h incubation. Recovery of the disruption was observed for $0 \mathrm{~h}$, $24 \mathrm{~h}$. Each assay was performed at least three times.

\section{Flow cytometric analysis}

For apoptosis and cell-cycle assay, cells were transfected with siTUG1, then treated with drugs for $24 \mathrm{~h}$ or not before collected. Cell apoptosis assay was conducted by using AnnexinV/propidium iodide detection kit (Keygene, Nanjing, China). For cell-cycle assay, cells were collected and fixed in $70 \%$ ethanol at $4{ }^{\circ} \mathrm{C}$ for $16 \mathrm{~h}$ and then stained with propidium iodide.

\section{Tumor xenograft experiments}

This study was conducted according to the institutional guidelines of Guangdong Province and were approved by the institutional guidelines of Guangdong Province and by the Use Committee for Animal Care. Male BALB/c nude mice aged 3-4 weeks were purchased from the Experimental Animal Center of Sun Yat-sen University (Guangzhou, China). Cells were harvested and re-suspended in serum free medium at a concentration of $1 \times 10^{7}$ cells $/ 0.2 \mathrm{ml}$. Each mouse was inoculated subcutaneously in the right flank with SCLC cells stably transduced with shTUG1 or shControl. Tumor size was monitored every 3 days, and mice were euthanized after 4 weeks. In vivo chemosensitivity assays, the animals were treated with chemotherapeutics or PBS via intraperitoneal injection $(7 \mathrm{mg} / \mathrm{kg}$ body weight etoposide [once every 2 days] and $3 \mathrm{mg} / \mathrm{kg}$ body weight cisplatin [once every 8 days]).

\section{Western blotting}

Equivalent amounts of cell protein lysates were electrophoresed on an 10\% SDS-polyacrylamide gel, transferred to a PVDF membrane. Then the membrane was incubated with primary antibodies overnight at $4{ }^{\circ} \mathrm{C}$. Followed incubated by horseradish peroxidase-labeled secondary antibody. Anti- 
LIMK2b was purchased from Abcam (1:1,000). GAPDH was used as a protein-loading control. The immune complexes were detected by chemiluminescence (ECL).

\section{RNA immunoprecipitation (RIP) assay}

RNA immunoprecipitation was conducted using Magna RIP RNA-Binding Protein Immunoprecipitation Kit (Millipore) following the manufacturer's protocol.

\section{Chromatin immunoprecipitation}

The ChIP assays were performed according to the Protocol for the fast chromatin immunoprecipitation (ChIP) method [42]. EZH2 antibody was purchased from Abcam. H3 trimethyl Lys 27 antibody was from Millipore. Gene specific primers for LIMK2b are listed in Additional file 5: Table S5. Results were normalized using the internal control IgG. Precipitated chromatin DNA was recovered and analyzed by qPCR.

\section{Statistical analysis}

Statistical analyses were performed using SPSS version 21.0 software. Experimental results are presented using means \pm SD. Independent- samples $T$ test or one-way ANOVA were used to analyze the possible differences between groups. The association between TUG1 expression and clinical features were analyzed by Pearson Chi-Square test. Survival curves were assessed by Kaplain-Meier analysis. Prognostic factors were analyzed by univariate and multivariate analyses (Cox proportional hazards model). $\mathrm{P}$ values $<0.05$ was considered statistically significant.

\section{Additional files}

Additional file 1: Figure S1. Relative expression level of TUG1 or EZH2 in H69, H446, H69AR and H446DDP cells transfected with siNC or si-TUG1 or si-EZH2. ${ }^{*}, P<0.05 ;{ }^{* *}, P<0.001$. (TIF $3849 \mathrm{~kb}$ )

Additional file 2: Figure S2. TUG1 promoted migration and invasion of SCLC cells in vitro. (A) Cell migration was quantified by wound healing assay. Cells were imaged immediately ( $0 \mathrm{~h}$ ) and $24 \mathrm{~h}$ after scratches were created to measure the percentage of wound healed area. Representative images at different time points are shown. (B) Cell morphology graph of invasive cells in H446, H69AR and H446DDP cells after stable transfection of shTUG1 or shControl. Data represent mean \pm SD of three independent experiments. (TIF $5658 \mathrm{~kb}$ )

Additional file 3: Figure S3. Cell apoptosis and cell cycle were assayed by flow cytometric analysis after $\mathrm{H} 69$ and $\mathrm{H} 446$ cells were transfected with siTUG1. (TIF $6255 \mathrm{~kb}$ )

Additional file 4: Figure S4. Apoptosis of H69AR-siTUG1, H446DDP-siTUG1 cells induced by anticancer drugs was significantly increased compared with controls. (TIF $6733 \mathrm{~kb}$ )

Additional file 5: Table S5. aRT-PCR primers. (XLS $20 \mathrm{~kb}$ )

\section{Abbreviations}

ADM: Adriamycin; CCK-8: Cell counting kit-8 assay; ChIP: Chromatin immunoprecipitation; DDP: Cisplatin; IncRNAs: Long non-coding RNAs; PRC2: Polycomb repressive complex2; qRT-PCR: Quantitative real-time polymerase chain reaction; RIP: RNA Binding protein immunoprecipitation; SCLC: Small cell lung cancer; VP-16: Etoposide

\section{Acknowledgements}

Disclosure of potential conflicts of interest.

There are no potential conflicts of interest to disclose.

\section{Funding}

This work was supported by the National Natural Science Foundation of China (81172241) and Natural Science Foundation of Guangdong Province (key) (2015A030311028)

Availability of data and materials Not applicable.

\section{Author contributions}

GL and NY conceived and designed the experiments. NY, Ma F and HW performed the experiments. NY, Ma F, FS and LM analyzed the data. NY, Ma F and WT wrote the paper. The first two authors contribute equally to this paper. All authors read and approved the final manuscript.

\section{Competing interests}

The authors declare that they have no competing interests.

\section{Consent for publication}

No conflict of interest exits in the submission of this manuscript, and manuscript is approved by all authors for publication.

\section{Ethics approval and consent to participate}

Under the protocol approved by the Institutional Review Board, informed consent was obtained from the patients or their guardians. The Ethical Committee of The First Affiliated Hospital of Hebei North University approved the tissue collection and studies with collected tumor tissues. All procedures involving animals were performed according to the guidelines of the Association for the Assessment and Accreditation of Laboratory Animal Care International.

\section{Author details}

'Department of Pathology Zhujiang Hospital, Southern Medical University, 253 Gongye Road, Guangzhou 510282, People's Republic of China. ${ }^{2}$ Department of Oncology, The First Affiliated Hospital of Hebei North University, Zhangjiakou, China. ${ }^{3}$ Department of Oncology, Zhujiang Hospital, Southern Medical University, Guangzhou, China.

Received: 24 May 2016 Accepted: 19 December 2016

Published online: 09 January 2017

\section{References}

1. Haddadin S, Perry MC. History of small-cell lung cancer. Clin Lung Cancer. 2011;12(2):87-93.

2. Hann CL, Rudin CM. Management of small-cell lung cancer: incremental changes but hope for the future. Oncology (Williston Park). 2008;22(13): 1486-92.

3. Riaz SP, Luchtenborg M, Coupland VH, Spicer J, Peake MD, Moller H. Trends in incidence of small cell lung cancer and all lung cancer. Lung Cancer-J laslc. 2012;75(3):280-4.

4. Murray N, Turrisi AR. A review of first-line treatment for small-cell lung cancer. J Thorac Oncol. 2006;1(3):270-8.

5. Sarvi S, Mackinnon AC, Avlonitis N, et al. CD133+ cancer stem-like cells in small cell lung cancer are highly tumorigenic and chemoresistant but sensitive to a novel Neuropeptide antagonist. Cancer Res. 2014:74(5):1554-65.

6. Mountzios G, Dimopoulos MA, Soria JC, Sanoudou D, Papadimitriou CA. Histopathologic and genetic alterations as predictors of response to treatment and survival in lung cancer: a review of published data. Crit Rev Oncol Hematol. 2010;75(2):94-109.

7. Ulitsky I, Bartel DP. LincRNAs: genomics, evolution, and mechanisms. Cell. 2013;154(1):26-46.

8. Batista PJ, Chang HY. Long noncoding RNAs: cellular address codes in development and disease. Cell. 2013;152(6):1298-307.

9. Mercer TR, Dinger ME, Mattick JS. Long non-coding RNAs: insights into functions. Nat Rev Genet. 2009;10(3):155-9.

10. Kung JT, Colognori D, Lee JT. Long noncoding RNAs: past, present, and future. Genetics. 2013;193(3):651-69. 
11. He $Y$, Meng $X M$, Huang $C$, et al. Long noncoding RNAs: Novel insights into hepatocelluar carcinoma. Cancer Lett. 2014;344(1):20-7.

12. Chakravarty D, Sboner A, Nair SS, et al. The oestrogen receptor alpharegulated IncRNA NEAT1 is a critical modulator of prostate cancer. Nat Commun. 2014;5:5383.

13. Huang MD, Chen WM, Qi FZ, et al. Long non-coding RNA ANRIL is upregulated in hepatocellular carcinoma and regulates cell apoptosis by epigenetic silencing of KLF2. J Hematol Oncol. 2015;8:50.

14. Li Z, Zhao X, Zhou Y, et al. The long non-coding RNA HOTTIP promotes progression and gemcitabine resistance by regulating HOXA13 in pancreatic cancer. J Transl Med. 2015;13:84.

15. Gutschner T, Hammerle M, Eissmann M, et al. The noncoding RNA MALAT1 is a critical regulator of the metastasis phenotype of lung cancer cells. Cancer Res. 2013;73(3):1180-9.

16. Braconi $\mathrm{C}$, Kogure $\mathrm{T}$, Valeri $\mathrm{N}$, et al. MicroRNA-29 can regulate expression of the long non-coding RNA gene MEG3 in hepatocellular cancer. Oncogene. 2011:30(47):4750-6.

17. Yang $X$, Song $J H$, Cheng $Y$, et al. Long non-coding RNA HNF1A-AS1 regulates proliferation and migration in Oesophageal adenocarcinoma cells. Gut. 2014;63(6):881-90.

18. Fang $\mathrm{S}, \mathrm{Gao} H$, Tong $\mathrm{Y}$, et al. Long noncoding RNA-HOTAIR affects chemoresistance by regulating HOXA1 methylation in small cell lung cancer cells. Lab Invest. 2016;96(1):60-8.

19. Young TL, Matsuda T, Cepko CL. The noncoding RNA taurine upregulated gene 1 is required for differentiation of the murine retina. Curr Biol. 2005;15(6):501-12

20. Ma B, Li M, Zhang L, et al. Upregulation of long non-coding RNA TUG1 correlates with poor prognosis and disease status in osteosarcoma. Tumour Biol. 2015.

21. Han Y, Liu Y, Gui Y, Cai Z. Long intergenic non-coding RNA TUG1 is overexpressed in urothelial carcinoma of the bladder. J Surg Oncol. 2013;107(5):555-9.

22. Xu Y, Wang J, Qiu M, et al. Upregulation of the long noncoding RNA TUG1 promotes proliferation and migration of esophageal squamous cell carcinoma. Tumour Biol. 2015;36(3):1643-51.

23. Khalil AM, Guttman $M$, Huarte $M$, et al. Many human large intergenic noncoding RNAs associate with chromatin-modifying complexes and affect gene expression. Proc Natl Acad Sci U S A. 2009;106(28):11667-72.

24. Zhang EB, Yin DD, Sun $M$, et al. P53-regulated long non-coding RNA TUG1 affects cell proliferation in human non-small cell lung cancer, partly through epigenetically regulating HOXB7 expression. Cell Death Dis. 2014:5:e1243.

25. Zhang E, He X, Yin D, et al. Increased expression of long noncoding RNA TUG1 predicts a poor prognosis of gastric cancer and regulates cell proliferation by epigenetically silencing of p57. Cell Death Dis. 2016;7:e2109.

26. Orom UA, Derrien $T$, Beringer $M$, et al. Long noncoding RNAs with enhancer-like function in human cells. Cell. 2010;143(1):46-58

27. Huang MD, Chen WM, Qi FZ, et al. Long non-coding RNA TUG1 is upregulated in hepatocellular carcinoma and promotes cell growth and apoptosis by epigenetically silencing of KLF2. Mol Cancer. 2015;14:165.

28. Acevedo K, Moussi N, Li R, Soo P, Bernard O. LIM kinase 2 is widely expressed in all tissues. J Histochem Cytochem. 2006;54(5):487-501.

29. Smolich B, Vo M, Buckley S, Plowman G, Papkoff J. Cloning and biochemical characterization of LIMK-2, a protein kinase containing two LIM domains. J Biochem. 1997;121(2):382-8.

30. Nomoto S, Tatematsu Y, Takahashi T, Osada H. Cloning and characterization of the alternative promoter regions of the human LIMK2 gene responsible for alternative transcripts with tissue-specific expression. Gene. 1999;236(2):259-71.

31. Vlecken DH, Bagowski CP. LIMK1 and LIMK2 are important for metastatic behavior and tumor cell-induced angiogenesis of pancreatic cancer cells. Zebrafish. 2009;6(4):433-9.

32. Scott $G A, M c C l e l l a n d ~ L A$, Fricke $A F$, Fender A. Plexin C1, a receptor for semaphorin 7a, inactivates cofilin and is a potential tumor suppressor for melanoma progression. J Invest Dermatol. 2009;129(4):954-63.

33. Suyama E, Wadhwa R, Kawasaki $H$, et al. LIM kinase-2 targeting as a possible anti-metastasis therapy. J Gene Med. 2004;6(3):357-63.

34. Dan S, Tsunoda T, Kitahara O, et al. An integrated database of chemosensitivity to 55 anticancer drugs and gene expression profiles of 39 human cancer cell lines. Cancer Res. 2002;62(4):1139-47.

35. Gamell C, Schofield AV, Suryadinata R, Sarcevic B, Bernard O. LIMK2 mediates resistance to chemotherapeutic drugs in neuroblastoma cells through regulation of drug-induced cell cycle arrest. PLoS One. 2013;8(8):e72850.

36. Osada H, Hasada K, Inazawa J, et al. Subcellular localization and protein interaction of the human LIMK2 gene expressing alternative transcripts with tissue-specific regulation. Biochem Biophys Res Commun. 1996;229(2):582-9.
37. Ikebe C, Ohashi K, Fujimori T, et al. Mouse LIM-kinase 2 gene: cDNA cloning, genomic organization, and tissue-specific expression of two alternatively initiated transcripts. Genomics. 1997;46(3):504-8.

38. Hsu FF, Lin TY, Chen JY, Shieh SY, p53-mediated transactivation of LIMK2b links actin dynamics to cell cycle checkpoint control. Oncogene. 2010; 29(19):2864-76.

39. Ku M, Koche RP, Rheinbay E, et al. Genomewide analysis of PRC1 and PRC2 occupancy identifies two classes of bivalent domains. PLoS Genet. 2008; 4(10):e1000242

40. Margueron R, Reinberg D. The polycomb complex PRC2 and its mark in life Nature. 2011:469(7330):343-9.

41. Tsang DP, Cheng AS. Epigenetic regulation of signaling pathways in cancer: role of the histone methyltransferase EZH2. J Gastroenterol Hepatol. 2011; 26(1):19-27

42. Nelson JD, Denisenko O, Bomsztyk K. Protocol for the fast chromatin immunoprecipitation (ChIP) method. Nat Protoc. 2006;1(1):179-85.

\section{Submit your next manuscript to BioMed Central and we will help you at every step:}

- We accept pre-submission inquiries

- Our selector tool helps you to find the most relevant journal

- We provide round the clock customer support

- Convenient online submission

- Thorough peer review

- Inclusion in PubMed and all major indexing services

- Maximum visibility for your research

Submit your manuscript at www.biomedcentral.com/submit
) Biomed Central 